\title{
Joint time-state generalized semiconcavity of the value function of a jump diffusion optimal control problem
}

\author{
Ermal Feleqi
}

\begin{abstract}
We prove generalized semiconcavity results, jointly in time and state variables, for the value function of a stochastic finite horizon optimal control problem, where the evolution of the state variable is described by a general stochastic differential equation (SDE) of jump type. Assuming that terms comprising the SDE are $C^{1}$-smooth, and that running and terminal costs are semiconcave in generalized sense, we show that the value function is also semiconcave in generalized sense, estimating the semiconcavity modulus of the value function in terms of smoothness and generalized semiconcavity moduli of data. Of course, these translate into analogous regularity results for (viscosity) solutions of integro-differential Hamilton-Jacobi-Bellman equations due to their controllistic interpretation. This paper may be seen as a sequel to Feleqi (Dyn Games Appl $3(4): 523-536,2013)$, where we dealt with the generalized semiconcavity of the value function only in the state variable.
\end{abstract}

Mathematics Subject Classification. 35D10, 35E10, 60H30, 93E20.

Keywords. Generalized semiconcavity, Value function, Optimal control, Jump diffusions, Partial integro-differential Hammilton-Jacobi-Bellman equations.

\section{Introduction}

In this article we continue our work initiated in [26] on establishing generalized semiconcavity results for the value function of a finite horizon jump diffusion optimal control problem. While in [26] we dealt with the problem of obtaining generalized semiconcavity estimates for the value function in the state variable, uniformly in time, here we prove generalized semiconcavity results in time and state variables jointly.

Under appropriate assumptions on the data - which follow from those made in this paper - the value function can be interpreted as the unique vis- 
cosity solution with at most polynomial growth of a partial integro-differential equation of Hamilton-Jacobi-Bellman (abbr. HJB) type

$$
\left\{\begin{aligned}
& \frac{\partial u}{\partial t}+\inf _{\alpha \in A}\left\{L(t, x, \alpha)+b(t, x, \alpha) \cdot \nabla u+\frac{1}{2} \operatorname{tr}\left(\sigma(t, x, \alpha) \sigma^{t}(t, x, \alpha) D^{2} u\right)\right. \\
&+\int_{E}(u(\cdot, \cdot+H(t, x, z, \alpha))-u-\nabla u \cdot H(t, x, z, \alpha)) \nu(d z) \\
&\left.+\int_{E^{c}}(u(\cdot, \cdot+K(t, x, z, \alpha))-u) \nu(d z)\right\}=0 \quad \text { in }[0, T) \times \mathbb{R}^{d} \\
& u(T, \cdot)=\psi \quad \text { in } \mathbb{R}^{d},
\end{aligned}\right.
$$

where $T>0, \nu$ is a Lévy measure on $\mathbb{R}^{d}, d \in \mathbb{N}, \mathbb{R}^{d} \backslash\{0\}=E \cup E^{c}$ for some set $E$ such that $E \cup\{0\}$ is open and bounded in $\mathbb{R}^{d}, A$ is some metric space - to be interpreted as the set of controls - and $b, \sigma, H, K, L, \psi$ are given maps as in (2.2) below. In addition, we assume that the Lévy measure $\nu$ is finite, that is, $\nu\left(\mathbb{R}^{d} \backslash\{0\}\right)<\infty$. We make this assumption for technical reasons related to a affine change of the time variable in stochastic integrals with respect to a Poisson measure, which requires a change of probability measure called Kulik's transformation [for precise definitions, see (3.8) and (3.9) below; for the definition of the "new" probability measure we have to assume that $\left.\nu\left(\mathbb{R}^{d} \backslash\{0\}\right)<\infty\right]$. We do not know whether our results extend to a general Lévy measure or not. The case of classical semiconcavity (linear modulus) has been treated in [33], where the author assumed $\nu\left(\mathbb{R}^{d} \backslash\{0\}\right)<\infty$ as well. So the extension of the results of [33] and of this paper to a general Lévy measure remains an open problem.

Before stating results we recall the following

Definition 1.1. [21] Given an upper semicontinuous nondecreasing function $\omega: \mathbb{R}_{+} \rightarrow \mathbb{R}_{+}$such that $\omega(0+)=\lim _{\rho \rightarrow 0+} \omega(\rho)=0$ (such a function is called a semiconcavity modulus), we say that a function $u: K \rightarrow \mathbb{R}$, where $K$ is some subset of $\mathbb{R}^{d}$, is an $\omega$-semiconcave function if

$$
\begin{aligned}
& \lambda u\left(x_{1}\right)+(1-\lambda) u\left(x_{2}\right)-u\left(\lambda x_{1}+(1-\lambda) x_{2}\right) \\
& \quad \leq \lambda(1-\lambda)\left|x_{1}-x_{2}\right| \omega\left(\left|x_{1}-x_{2}\right|\right)
\end{aligned}
$$

for all $x_{1}, x_{2} \in K$ such that the segment $\left[x_{1}, x_{2}\right] \subset K$ and $0 \leq \lambda \leq 1$. A function $u$ is called $\omega$-semiconvex if $-u$ is $\omega$-semiconcave. We say that $u$ is of class $C^{1, \omega}$ or $C^{1, \omega}$-regular if it is both $\omega$-semiconcave and $\omega$-semiconvex. ${ }^{1}$

A vector-valued function $u: K \rightarrow \mathbb{R}^{d}$ is said to be of class $C^{1, \omega}$ (or, $C^{1, \omega}$-regular) if each of its components is of class $C^{1, \omega}$.

Our results go roughly as follows. Let $b, \sigma, H, K$ be, in order, of class $C^{1, \omega_{1}}, C^{1, \omega_{2}}, C^{1, \omega_{3}}, C^{1, \omega_{4}}$, respectively, and $L, \psi$ be $\omega_{5^{-}}$and $\omega_{6}$-semiconcave, jointly in time and state variables, uniformly in control and jump variables, where all the $\omega_{i}$ 's are given semiconcavity moduli. Assume also that all these maps are bounded, globally Lipschitz continuous in time and state variables, uniformly in control and jump variables, and that the Lévy measure is finite.

\footnotetext{
1 This definition is justified, e.g., by [21, Theorem 3.3.7, p. 60].
} 
Then, for all $\delta \in] 0, T]$, the unique viscosity solution $u$ of (1.1) with polynomial growth is $\omega$-semicocave on $[0, T-\delta] \times \mathbb{R}^{d}$ for some modulus $\omega$ that can be expressed in terms of the given moduli $\omega_{i}$ for $i=1, \ldots, 6$ (for precise results see Theorems 2.2 and 2.11 ). One cannot hope to prove $\omega$-semiconcavity of $u$ on all of $[0, T] \times \mathbb{R}^{d}$ for it would imply the Lipschitz continuity of $u$ on bounded subsets of $[0, T] \times \mathbb{R}^{d}$, which is known to be not true in general as shown by the simple Example 3.1 in [11].

The results of the present paper are (to the best of our knowledge) new for two reasons: firstly, because the semiconcavity moduli considered here are quite general (in contrast to the usual linear moduli, corresponding to classical semiconcavity); part of these regularity results may be new even for "pure" diffusion processes $(H=K=0)$, if not also for the "deterministic" processes ( $\sigma=H=K=0)$; secondly, because the results are given for general possibly degenerate jump SDEs (in the literature one usually considers continuous diffusions or jump diffusion with some kind of ellipticity hypothesis).

The role of semiconcavity in first-order Hamilton-Jacobi-Bellman equations and optimal control was pointed out by Kružkov [37-39] (see also [8,35]) and in connections with viscosity solutions in $[19,20,22-24,52]$. For secondorder HJB equations the first one-sided estimates for second difference quotients (or semiconcavity estimates with a linear modulus) were obtained in [40, 51]. For more results and extensive historical and bibliographical remarks, not only on semiconcavity estimates, but, more generally, on optimal control and HJB equations, the reader is referred to books $[2,21,27,54,56]$. Semiconcavity estimates can also be proved via PDE techniques (comparison principles) as in $[29,31]$.

There has been considerable work recently regarding (classical) semiconcavity in time-space for the value functions of optimal control problems for diffusions with or without jumps, or, equivalently, viscosity solutions of P(I)DEs of HJB type. The case of diffusions without jumps $(H=K=0)$, that is, of second-order PDEs of HJB type, is treated in [11] and in [12]. The case of general PIDEs of HJB type is treated in [33], but, as we said, under the restrictive assumption that the Lévy measure $\nu$ should be finite; the case of a general Lévy measure (for which $\int_{\mathbb{R}^{d}} \min \left\{1,|z|^{2}\right\} \nu(d z)<\infty$ ), according to [33], is still open. Moreover, Lipschitz and semiconcavity estimates were proved also in [53] by PDE techniques for a wide class of PDIEs not necessarily arising from optimal control problems.

Related to our discussion is also [9], which addresses convexity preservation results (in space variable) for HJB PIDEs, and their significance to financial applications.

Of course, we are not aware of any systematic treatment dedicated to generalized semiconcavity in the sense of Definition 1.1 in both time and space variables jointly. Our paper [26] was probably the first to deal systematically with the issue of establishing generalized semiconcavity results for the value function in the state variable, uniformly in time, and, the present paper should be seen as a sequel to that one. 
The results of this paper are a (small) part of the vast regularity theory of PIDEs. First results on this subject were obtained assuming nondegenerate diffusions or elliptic second-order differential (local) terms as in $[7,28,30]$ (just to mention a very few references without any pretense of completeness) and references therein. Recently, there has been a revival of interest on the theory of PIDEs which is due to the work on one hand of Caffarelli et al. [13-18], and Barles, Chasseigne, Ciomaga, Imbert on the other [4-6]. These authors, differently from the earlier ones, prove regularity results, such as Hölder, Lipschitz, $C^{1, \alpha}$-estimates, under a kind of ellipticity assumption, which is not any more due to the second-order local terms (or to the presence of nonsingular diffusions), but comes either from the nonlocal terms or from the combined effect of both local and nonlocal terms. Related results have been obtained by other authors as well $[10,25,32,34,36,44,45,49,50]$.

Our interest in the regularity theory of partial integro-differential equations of HJB type and related optimal control problems arose from the recent theory of Mean Field Games (abbr. MFG) developed by Lasry and Lions [4648], which yields limiting models for symmetric, non-zero sum, non-cooperative $N$-player games with the interaction between the players being of mean-field type. It is of interest to study MFG models where the dynamic of an average or representative agent is a jump diffusion because stochastic phenomena in Economics and Finance applications exhibit jumps and other deviations from pure diffusions. The MFG paradigm would lead in this case to PIDEs of HJB for the optimal values of the average agents coupled with Fokker-Planck PIDEs for probability distributions of their optimal dynamics. To our knowledge the study of such systems of PIDEs remains largely to be done. In particular, we are interested in extending to these systems of PIDES our results in $[3,26]$.

The proof is based on interpreting the said solution of (1.1) as the value function of a stochastic optimal control problem for jump diffusion processes, that is, processes which are solutions of appropriate stochastic differential equations of jump type driven by Brownian motions and Poisson random measures independent of each other (abbr. SDEs) see, e.g., [54] and references therein. Furthermore, we rely on the method of affine time changes for Brownian motions as in [11,12] and for Poisson random measures as in [33]. While the corresponding change of variable formula for Wiener integrals is rather easy, for stochastic integrals with respect to Poisson random measures, the formula is more involved and requires a change of probability on the underlying sample space via the so called Kulik's transformation; see [33] for more details and references. Other tools are Burkholder type inequalities as stated for example in [43], and of course Gronwall's inequality.

The paper is organized as follows. Main results (Theorems 2.2 and 2.11) are stated in the next section. The proof of technical lemmas is postponed to the "Appendix" (Sect. 3) in order to ensure a better readability of the paper.

Notation In accordance with common practice, we usually use the same letter (here $C_{\delta}$ ) to denote possibly different constants in a chain of estimates/inequalities, which however depend only on the same data; see, e.g., the proofs of Lemmas 2.5 and 2.7 . 


\section{The optimal control of jump diffusions}

Let $T>0$ be a fixed time horizon, $A$ a metric space - to be interpreted as the set of control values -,$\nu$ a finite Lévy measure on $Z=\mathbb{R}^{d} \backslash\{0\}$ and $Z=E \cup E^{c}$, where $E$ is a subset of $\mathbb{R}^{d} \backslash\{0\}$ such that $E \cup\{0\}$ is a bounded open subset of $\mathbb{R}^{d}$. For all $s \in[0, T]$ we denote by $\mathcal{R}_{s}$ the collection of the following entities ${ }^{2}$

$$
R=\left(\Omega, \mathcal{F}, \mathbb{F}=\left(\mathcal{F}_{t}\right)_{s \leq t \leq T}, \mathbb{P}, W(\cdot), N=N(d t d z)\right),
$$

that satisfy the following conditions:

$$
\left(\Omega, \mathcal{F}, \mathbb{F}=\left(\mathcal{F}_{t}\right)_{s \leq t \leq T}, \mathbb{P}\right)
$$

is a complete filtered probability space such that the filtration $\mathbb{F}$ satisfies the usual hypotheses (that is, $\mathbb{F}$ is right continuous and every sub- $\sigma$ algebra $\mathcal{F}_{t}$, for $0 \leq t \leq T$, is complete with respect to the probability measure $\mathbb{P}$;

- $W=W(\cdot)$ is a standard $m$-dimensional $(m \in \mathbb{N}) \mathbb{F}$-adapted Brownian motion on $(\Omega, \mathcal{F}, \mathbb{P})$;

- $N=N(d t d z)$ is a $\mathbb{F}$-adapted Poisson random measure on $\mathbb{R}^{+} \times Z$ and on probability space $(\Omega, \mathcal{F}, \mathbb{P})$ with intensity measure $\nu$ on $Z$, and with associated compensator $\tilde{N}=\tilde{N}(d t d z)=N(d t d z)-d t \nu(d z)$;

- $W$ and $N$ are independent of each-other and moreover have increments that are independent of the filtration $\mathbb{F}$, that is, $W\left(t_{2}\right)-W\left(t_{1}\right), N\left(t_{2}\right)-$ $N\left(t_{1}\right)$ are independent of $\mathcal{F}_{t_{1}}$ for all $s \leq t_{1} \leq t_{2} \leq T$.

Let

$$
\begin{aligned}
& b:[0, T] \times \mathbb{R}^{d} \times A \rightarrow \mathbb{R}^{d}, \quad \sigma:[0, T] \times \mathbb{R}^{d} \times A \rightarrow \mathbb{R}^{d \times m}, \\
& H:[0, T] \times \mathbb{R}^{d} \times E \times A \rightarrow \mathbb{R}^{d}, \quad K:[0, T] \times \mathbb{R}^{d} \times E^{c} \times A \rightarrow \mathbb{R}^{d}, \\
& L:[0, T] \times \mathbb{R}^{d} \times A \rightarrow \mathbb{R}, \quad \psi: \mathbb{R}^{d} \rightarrow \mathbb{R}
\end{aligned}
$$

be (measurable) maps, $p \geq 2, C_{i}, L_{i} \geq 0$ fixed constants and $\omega_{i}$ regularity moduli, for $i=1, \ldots, 6$.

Assume that the following hold true:

(B) (bounded data)

(i) (bounded dynamics)

$$
\begin{aligned}
|b(r, x, \alpha)| & \leq C_{1}, & & |\sigma(r, x, \alpha)| \leq C_{2}, \\
|H(r, x, z, \alpha)| & \leq C_{3}, & & \left|K\left(r, x, z^{c}, \alpha\right)\right| \leq C_{4},
\end{aligned}
$$

(ii) (bounded costs)

$$
|L(r, x, \alpha)| \leq C_{5}, \quad|\psi(x)| \leq C_{6}
$$

(L) (Lipschitz continuous data)

(i) (Lipschitz continuous dynamics)

$$
\left|b\left(r_{1}, x_{1}, \alpha\right)-b\left(r_{2}, x_{2}, \alpha\right)\right| \leq L_{1}\left(\left|r_{1}-r_{2}\right|+\left|x_{1}-x_{2}\right|\right) \text {, }
$$

\footnotetext{
${ }^{2}$ Which we could call probability references if we were to adapt a terminology analogous to the one adapted in [27].
} 


$$
\begin{aligned}
& \left|\sigma\left(r_{1}, x_{1}, \alpha\right)-\sigma\left(r_{2}, x_{2}, \alpha\right)\right| \leq L_{2}\left(\left|r_{1}-r_{2}\right|+\left|x_{1}-x_{2}\right|\right), \\
& \int_{E}\left|H\left(r_{1}, x_{1}, z, \alpha\right)-H\left(r_{2}, x_{2}, z, \alpha\right)\right|^{p} \nu(d z) \leq L_{3}^{p}\left(\left|r_{1}-r_{2}\right|+\left|x_{1}-x_{2}\right|\right)^{p}, \\
& \int_{E^{c}}\left\|K\left(r_{1}, x_{1}, z, \alpha\right)-K\left(r_{2}, x_{2}, z, \alpha\right)\right\|^{p} \nu(d z) \leq L_{4}^{p}\left(\left|r_{1}-r_{2}\right|+\left|x_{1}-x_{2}\right|\right)^{p},
\end{aligned}
$$

(ii) (Lipschitz continuous costs)

$$
\begin{aligned}
\left|L\left(r_{1}, x_{1}, \alpha\right)-L\left(r_{2}, x_{2}, \alpha\right)\right| & \leq L_{5}\left(\left|r_{1}-r_{2}\right|+\left|x_{1}-x_{2}\right|\right), \\
\left|\psi\left(x_{1}\right)-\psi\left(x_{2}\right)\right| & \leq L_{6}\left|x_{1}-x_{2}\right|,
\end{aligned}
$$

(S) (semiconcave data)

(i) $\left(C^{1, \omega}\right.$-regular dynamics $)$

$$
\begin{aligned}
& \left|\lambda b\left(r_{1}, x_{1}, \alpha\right)+(1-\lambda) b\left(r_{2}, x_{2}, \alpha\right)-b\left(r_{\lambda}, x_{\lambda}, \alpha\right)\right| \\
& \quad \leq \lambda(1-\lambda)\left(\left|r_{1}-r_{2}\right|+\left|x_{1}-x_{2}\right|\right) \omega_{1}\left(\left|r_{1}-r_{2}\right|+\left|x_{1}-x_{2}\right|\right), \\
& \left|\lambda \sigma\left(r_{1}, x_{1}, \alpha\right)+(1-\lambda) \sigma\left(r_{2}, x_{2}, \alpha\right)-\sigma\left(r_{\lambda}, x_{\lambda}, \alpha\right)\right| \\
& \quad \leq \lambda(1-\lambda)\left(\left|r_{1}-r_{2}\right|+\left|x_{1}-x_{2}\right|\right)|| \omega_{2}\left(\left|r_{1}-r_{2}\right|+\| x_{1}-x_{2}||\right), \\
& \int_{E}\left|\lambda H\left(r_{1}, x_{1}, z, \alpha\right)+(1-\lambda) H\left(r_{2}, x_{1}, z, \alpha\right)-H\left(r_{\lambda}, x_{\lambda}, z, \alpha\right)\right|^{p} \nu(d z) \\
& \quad \leq\left(\lambda(1-\lambda)\left(\left|r_{1}-r_{2}\right|+\left|x_{1}-x_{2}\right|\right) \omega_{3}\left(\left|r_{1}-r_{2}\right|+\left|x_{1}-x_{2}\right|\right)\right)^{p}, \\
& \int_{E^{c}}|| \lambda K\left(r_{1}, x_{1}, z^{c}, \alpha\right)+(1-\lambda) K\left(r_{2}, x_{1}, z^{c}, \alpha\right)-K\left(r_{\lambda}, x_{\lambda}, z^{c}, \alpha\right) \|^{p} \nu\left(d z^{c}\right) \\
& \quad \leq\left(\lambda(1-\lambda)\left(\left|r_{1}-r_{2}\right|+|| x_{1}-x_{2} \|\right) \omega_{4}\left(\left|r_{1}-r_{2}\right|+\left|x_{1}-x_{2}\right|\right)\right)^{p}
\end{aligned}
$$

(ii) ( $\omega$-semiconcave costs)

$$
\begin{aligned}
& \lambda L\left(r_{1}, x_{1}, \alpha\right)+(1-\lambda) L\left(r, x_{2}, \alpha\right)-L\left(r_{\lambda}, x_{\lambda}, \alpha\right) \\
& \quad \leq \lambda(1-\lambda)\left(\left|r_{1}-r_{2}\right|+\left|x_{1}-x_{2}\right|\right) \omega_{5}\left(\left|r_{1}-r_{2}\right|+\left|x_{1}-x_{2}\right|\right), \\
& \lambda \psi\left(x_{1}\right)+(1-\lambda) \psi\left(x_{2}\right)-\psi\left(x_{\lambda}\right) \leq \lambda(1-\lambda)\left|x_{1}-x_{2}\right| \omega_{6}\left(\left|x_{1}-x_{2}\right|\right)
\end{aligned}
$$

for all $r, r_{1}, r_{2} \in[0, T], x, x_{1}, x_{2} \in \mathbb{R}^{d}, 0 \leq \lambda \leq 1, \alpha \in A, z \in E, z^{c} \in E$, where $r_{\lambda}=\lambda r_{1}+(1-\lambda) r_{2}, x_{\lambda}=\lambda x_{1}+(1-\lambda) x_{2}$. Since $p \geq 2$ and $\nu(Z)<\infty$, then it follows that estimates for $H$ and $K$ hold also for $p=2$.

We cannot handle arbitrary moduli, therefore we have to make assumptions on the moduli as well. However, these assumptions are not very restrictive and are verified by the moduli appearing in most applications of interest. We should notice that in many cases we can replace the regularity or semiconcavity modulus of a map by a larger one so that it satisfy our assumptions. For alternative assumptions on the moduli see Theorem 2.11 below.

To begin with we make either one of the following assumptions on the moduli. 
(MP) (Power type moduli).

(i) (Moduli of the dynamics). We assume that

$$
\omega_{i}(\rho)=k_{i} \rho^{\alpha_{i}}, \quad \forall \rho \geq 0, i=1, \ldots, 4,
$$

for given $0<\alpha_{i}(\leq 1), k_{i} \geq 0$ and also that

$$
p \geq 2\left(1+\max \left\{\alpha_{1}, \alpha_{2}, \alpha_{3}, \alpha_{4}\right\}\right) .
$$

(ii) (Moduli of the costs). Furthermore, we assume that

$$
\omega_{i}(\rho)=k_{i} \rho^{\alpha_{i}}, \quad \forall \rho \geq 0, i=5,6,
$$

for given $0<\alpha_{i}(\leq 1), k_{i} \geq 0$ and also that

$$
p \geq 1+\max \left\{\alpha_{5}, \alpha_{6}\right\} .
$$

Alternatively, we assume the following hold true.

(MC) (Moduli with concavity properties).

(i) (Moduli of the dynamics). The functions

$$
\begin{aligned}
\gamma_{i}(\rho) & =\left(\rho^{\beta_{i}} \omega_{i}^{2}(\rho)\right)^{q_{i}}, \quad \forall \rho \geq 0, i=1, \ldots, 4 \text { and given } 0 \leq \beta_{i} \leq 2, \\
1 & \leq q_{i} \leq \infty,
\end{aligned}
$$

are concave and, if $r_{i} \geq 1$ are such that $r_{i}^{-1}+q_{i}^{-1}=1$ for all $1=1, \ldots 4$, we assume also that

$$
1 \leq\left(2-\beta_{i}\right) r_{i} \leq p \quad \text { for } i=1, \ldots, 4 .
$$

(ii) (Moduli of the costs). Furthermore, the functions

$\gamma_{i}(\rho)=\left(\rho^{\beta_{i}} \omega_{i}(\rho)\right)^{q_{i}}, \quad \forall \rho \geq 0, i=5,6$, and given $0 \leq \beta_{i} \leq 1,1 \leq q_{i} \leq \infty$, are concave and, if $r_{i} \geq 1$ are such that $r_{i}^{-1}+q_{i}^{-1}=1$ for all $i=5,6$, then we assume also that

$$
1 \leq\left(1-\beta_{i}\right) r_{i} \leq p \quad \text { for } i=5,6 .
$$

Remark 2.1. The larger the $p$ is the more restrictive these assumptions become, so we aim at proving results for $p \geq 2$ as small as possible. In the case of (MP), by (2.3), (2.4), it suffices to assume that the above estimates (L), (S) hold true for $p=4$. as it is done in [33], where the case of classical semiconcavity estimates (that is, $\omega$-semiconcavity estimates with linear $\omega$-s) is treated. Indeed, it is not reasonable to take $\alpha_{i}>1(i=1, \ldots, 6)$, that is, a superlinear modulus, otherwise, by [21, Theorem 2.1.9], an $\omega$-semiconcave function would just be concave and a $C^{1, \omega}$ map would just be constant. In such a case one may just take $\omega_{i}=0$, that is, $k_{i}=0$ and $\alpha_{i}=0$. Still we cannot assume $p=2$ unless our results trivialize for this would force us to take $\alpha_{i}=0$ for all $i=1, \ldots, 4$.

For any $s \in[0, T], R \in \mathcal{R}_{s}$ as in (2.1), we consider the following optimal control problem:

(admissible controls) we take as set of admissible controls $\mathcal{A}_{R}(s, T)$ the set of $R$-predictable ${ }^{3}$ processes $\alpha(\cdot):[0, T] \rightarrow A$;

3 That is, predictable with respect to the filtration $\mathbb{F}$ of $R$. 
(controlled system) for any $x^{0} \in \mathbb{R}^{d}, \alpha(\cdot) \in \mathcal{A}_{R}(s, T)$ we consider the stochastic differential equation of jump type: for all $s \leq t \leq T$

$$
\begin{aligned}
x(t)= & x^{0}+\int_{s}^{t} b(r, x(r-), \alpha(r)) d r+\int_{s}^{t} \sigma(r, x(r-), \alpha(r)) d W(r) \\
& +\int_{s}^{t} \int_{E} H(r, x(r-), z, \alpha(r)) \tilde{N}(d r d z) \\
& +\int_{s}^{t} \int_{E^{c}} K(r, x(r-), z, \alpha(r)) N(d r d z)
\end{aligned}
$$

(cost functionals) for any $x^{0} \in \mathbb{R}^{d}, \alpha(\cdot) \in \mathcal{A}_{R}(s, T)$, if $x(\cdot)$ is the solution ${ }^{4}$ to (2.5), the cost is given by

$$
J_{R}\left(s, x^{0}, \alpha(\cdot)\right)=\mathbb{E}\left[\int_{s}^{T} L(t, x(t), \alpha(t)) d t+\psi(x(T))\right]
$$

(value function) the value function $V_{R}$ is given by

$$
V_{R}\left(s, x^{0}\right)=\inf _{\alpha(\cdot) \in \mathcal{A}_{R}(s, T)} J_{R}\left(s, x^{0}, \alpha(\cdot)\right),
$$

where, for each $\alpha(\cdot) \in \mathcal{A}_{R}(s, T), x(\cdot)$ is the solution of Eq. (2.5); we consider also

$$
V\left(s, x^{0}\right)=\inf _{R \in \mathcal{R}_{s}} V_{R}\left(s, x^{0}\right) .
$$

Under assumptions $(\mathrm{B}),(\mathrm{L}), V_{R}(s, \cdot)=V(s, \cdot)$ for all $s \in[0, T], R \in$ $\mathcal{R}_{s}$, and $V$ is actually the unique viscosity solutions of (1.1) with polynomial growth $[54,55]$. Actually, in [33] it is proved that $V$ is Lipschitz continuous on $[0, T-\delta] \times \mathbb{R}^{d}$ for any $\left.\left.\delta \in\right] 0, T\right]$.

As we pointed out in the introduction, $V$ is not in general locally Lipschitz continuous (and therefore not semiconcave in generalized sense) on $[0, T] \times \mathbb{R}^{d}$ (indeed $V(t, x)=\sqrt{T-t}$ for $b=0, \sigma=1, H=K=0, L=1, \psi=$ $0)$. However, we prove that, for every $0<\delta \leq T, V$ is $\omega$-semiconcave on $[0, T-\delta] \times \mathbb{R}^{d}$ for some modulus $\omega$ which can be expressed in terms of the moduli of the data of the problem.

Thus we fix also a $\delta \in] 0, T]$.

We prove the following generalized semiconcavity estimates in time-space.

Theorem 2.2. Assume (B), (L), $(S)$ and either $(M P)$ or $(M C)$. Then the value function $V$ is $\omega$-semiconcave on $[0, T-\delta] \times \mathbb{R}^{d}$ for some modulus $\omega$ of the form

$$
\omega(\rho)=\sum_{i=1}^{6} c_{i}^{\prime} \omega_{i}\left(c_{i} \rho\right)+c_{7} \rho \quad \forall \rho \geq 0
$$

for constants $c_{i}, c_{i}^{\prime} \geq 0$ for $i=1, \ldots, 6, c_{7} \geq 0$ that depend only on $d, m, T, \delta$, $\nu(Z), p, C_{i}, L_{i}$ for $i=1, \ldots, 6$.

\footnotetext{
${ }^{4}$ Under assumptions (B)-(i), (L)-(i), Eq. (2.5) is indeed uniquely solvable by a right continuous $\mathbb{R}^{d}$-valued process $x(\cdot)$ with left limits defined on $[s, T]$; in addition this process is $\mathbb{F}$-adapted; see [1, Theorems 6.2 .3 , p. 367 and 6.2 .9 , p. 374].
} 
In order to prove generalized semiconcavity estimates for $V$ on $[0, T-$ $\delta] \times \mathbb{R}^{d}$ (and in particular, Theorem 2.2 above) we take $s_{1}, s_{2} \in[0, T-\delta]$, $x_{1}^{0}, x_{2}^{0} \in \mathbb{R}^{d}, \lambda \in[0,1]$, and set $s_{\lambda}=\lambda s_{1}+(1-\lambda) s_{2}, x_{\lambda}^{0}=\lambda x_{1}^{0}+(1-\lambda) x_{2}^{0}$. Let $R \in \mathcal{R}_{s_{\lambda}}, \alpha(\cdot) \in \mathcal{A}_{R}\left(s_{\lambda}, T\right)$, and denote by $\tau_{1}, \tau_{2}$ the affine "time changes" that transform $\left[s_{1}, T\right]$, respectively, $\left[s_{2}, T\right]$, into $\left[s_{\lambda}, T\right]$, that is,

$$
\tau_{i}:\left[s_{i}, T\right] \rightarrow\left[s_{\lambda}, T\right], \quad \tau_{i}(t)=s_{\lambda}+\frac{T-s_{\lambda}}{T-s_{i}}\left(t-s_{i}\right) \quad \forall t \in\left[s_{i}, T\right], i=1,2,
$$

(which have derivatives $\left.\dot{\tau}_{i}=\left(T-s_{\lambda}\right) /\left(T-s_{i}\right)\right)$. We take

$$
R_{i}=\left(\Omega, \mathcal{F}_{i}, \mathbb{Q}_{i}, \mathbb{F}_{i}, \tau_{i}(W), \tau_{i}(N)\right)
$$

where $\mathbb{F}_{i}$ is defined as in (3.6) and $\mathbb{Q}_{i}$ as in (3.9). It is easy to see that $R_{i} \in \mathcal{R}_{s_{i}}$ and $\alpha_{i}(\cdot)=\alpha(\cdot) \circ \tau_{i} \in \mathcal{A}_{R_{i}}\left(s_{1}, T\right), i=1,2$.

Denoting by $x_{i}(\cdot)$ the solutions of Eq. (2.5) for $R=R_{i}, \alpha(\cdot)=\alpha_{i}(\cdot)$ and initial conditions $s=s_{i}, x^{0}=x_{i}^{0}$, for $i=1,2$, respectively; and by $x_{\lambda}(\cdot)$ the solution of (2.5) for the previously fixed $R \in \mathcal{R}_{s_{\lambda}}, \alpha(\cdot) \in \mathcal{A}_{R}\left(s_{\lambda}, T\right)$, initial conditions $s=s_{\lambda}, x^{0}=x_{\lambda}^{0}$, setting $\tilde{x}_{1}(\cdot)=x_{1}(\cdot) \circ \tau_{1}^{-1}, \tilde{x}_{2}(\cdot)=x_{2}(\cdot) \circ$ $\tau_{2}^{-1}, \tilde{x}_{\lambda}(\cdot)=\lambda \tilde{x}_{1}(\cdot)+(1-\lambda) \tilde{x}_{2}(\cdot)$, we obtain, by Burkholder inequalities and change of variable formulas for stochastic integrals with respect to affine time changes - see the detailed proof in the "Appendix" - the following estimates:

Lemma 2.3. For some $c \geq 0$, that depends only on $d, m, T, p, \nu(Z)$, and for every $t \in\left[s_{\lambda}, T\right]$,

$$
\begin{aligned}
& \mathbb{E} {\left[\left|\tilde{x}_{1}(t)-\tilde{x}_{2}(t)-x_{1}^{0}+x_{2}^{0}\right|^{p}\right] \leq c \int_{s_{\lambda}}^{t} \mathbb{E}\left[\mid \frac{1}{\dot{\tau}_{1}} b\left(r, \tilde{x}_{1}(r), \alpha(r)\right)\right.} \\
&\left.-\left.\frac{1}{\dot{\tau}_{2}} b\left(r, \tilde{x}_{2}(r), \alpha(r)\right)\right|^{p}\right] \\
&+c \int_{s_{\lambda}}^{t} \mathbb{E}\left[\left|\frac{1}{\sqrt{\dot{\tau}_{1}}} \sigma\left(r, \tilde{x}_{1}(r), \alpha(r)\right)-\frac{1}{\sqrt{\dot{\tau}_{2}}} \sigma\left(r, \tilde{x}_{2}(r), \alpha(r)\right)\right|^{p}\right] d r \\
&+c \int_{s_{\lambda}}^{t} \mathbb{E}\left[\int_{E} \mid\left(1-\frac{1}{\dot{\tau}_{1}}\right) H\left(r, \tilde{x}_{1}(r), z, \alpha(r)\right)\right. \\
&\left.-\left.\left(1-\frac{1}{\dot{\tau}_{2}}\right) H\left(r, \tilde{x}_{2}(r), z, \alpha(r)\right)\right|^{p} \nu(d z)\right] d r \\
&+c \int_{s_{\lambda}}^{t} \mathbb{E}\left[\int_{E^{c}}\left|K\left(r, \tilde{x}_{1}(r), z, \alpha(r)\right)-K\left(r, \tilde{x}_{2}(r), z, \alpha(r)\right)\right|^{p} \nu(d z)\right] d r \\
& \mathbb{E}\left[\left|\tilde{x}_{\lambda}(t)-x_{\lambda}(t)\right|^{2}\right] \\
& \leq c \int_{s_{\lambda}}^{t}\left[\mid \frac{\lambda}{\dot{\tau}_{1}} b\left(\tau_{1}^{-1}(r), \tilde{x}_{1}(r), \alpha(r)\right)+\frac{1-\lambda}{\dot{\tau}_{2}} b\left(\tau_{2}^{-1}(r), \tilde{x}_{2}(r), \alpha(r)\right)\right. \\
&\left.\quad-\left.b\left(r, x_{\lambda}(r), \alpha(r)\right)\right|^{2}\right] d r \\
& \quad+c \int_{s_{\lambda}}^{t} \mathbb{E}\left[\mid \frac{\lambda}{\dot{\tau}_{1}} \sigma\left(\tau_{1}^{-1}(r), \tilde{x}_{1}(r), \alpha(r)\right)+\frac{1-\lambda}{\dot{\tau}_{2}} \sigma\left(\tau_{2}^{-1}(r), \tilde{x}_{2}(r), \alpha(r)\right)\right.
\end{aligned}
$$




$$
\begin{aligned}
& \left.-\left.\sigma\left(r, x_{\lambda}(r), \alpha(r)\right)\right|^{2}\right] d r \\
& +c \int_{s_{\lambda}}^{t} \mathbb{E}\left[\int_{E} \mid \lambda H\left(\tau_{1}^{-1}(r), \tilde{x}_{1}(r), \alpha(r)\right)+(1-\lambda) H\left(\tau_{2}^{-1}(r), \tilde{x}_{2}(r), \alpha(r)\right)\right. \\
& \left.-\left.H\left(r, x_{\lambda}(r), \alpha(r)\right)\right|^{2} \nu(d z)\right] d r \\
& +c \int_{s_{\lambda}}^{t} \mathbb{E}\left[\int_{E^{c}} \mid \lambda\left(1-\frac{1}{\dot{\tau}_{1}}\right) K\left(\tau_{1}^{-1}(r), \tilde{x}_{1}(r), \alpha(r)\right)\right. \\
& \left.+(1-\lambda)\left(1-\frac{1}{\dot{\tau}_{1}}\right) K\left(\tau_{2}^{-1}(r), \tilde{x}_{2}(r), \alpha(r)\right)-\left.K\left(r, x_{\lambda}(r), \alpha(r)\right)\right|^{2} \nu(d z)\right] d r .
\end{aligned}
$$

For a better readability of the paper, the proof of this lemma and the others stated in this section is postponed to the "Appendix".

We need the following simple technical lemma which can be checked by straightforward computation, hence its proof, which in any case can be found in [11], is omitted.

Lemma 2.4. For any $0<\delta \leq T$ there exists $C_{\delta}>0$ such that

$$
\begin{aligned}
& \left|\tau_{1}^{-1}(r)-\tau_{2}^{-1}(r)\right|+\left|\frac{1}{\dot{\tau}_{1}}-\frac{1}{\dot{\tau}_{2}}\right|+\left|\frac{1}{\sqrt{\dot{\tau}_{1}}}-\frac{1}{\sqrt{\dot{\tau}_{2}}}\right| \leq C_{\delta}\left|s_{1}-s_{2}\right|, \\
& \lambda\left|1-\frac{1}{\sqrt{\dot{\tau}_{1}}}\right|+(1-\lambda)\left|1-\frac{1}{\sqrt{\dot{\tau}_{2}}}\right| \leq \frac{1}{2 \delta} \lambda(1-\lambda)\left|s_{1}-s_{2}\right|, \\
& \left|\lambda\left(1-\frac{1}{\sqrt{\dot{\tau}_{1}}}\right)+(1-\lambda)\left(1-\frac{1}{\sqrt{\dot{\tau}_{2}}}\right)\right| \leq \frac{1}{2 \delta^{2}} \lambda(1-\lambda)\left|s_{1}-s_{2}\right|
\end{aligned}
$$

for all $0 \leq s_{i} \leq T-\delta, i=1,2,0 \leq \lambda \leq 1, s \leq r \leq T$, where $s_{\lambda}=$ $\lambda s_{1}+(1-\lambda) s_{2}$. Moreover,

$$
\begin{aligned}
& \lambda \tau_{1}^{-1}(r)+(1-\lambda) \tau_{2}^{-1}(r)=r \\
& \lambda\left(1-\frac{1}{\dot{\tau}_{1}}\right)=-(1-\lambda)\left(1-\frac{1}{\dot{\tau}_{2}}\right)=\lambda(1-\lambda)\left(s_{1}-s_{2}\right)
\end{aligned}
$$

Lemma 2.5. (Lipschitz estimates in terms of initial conditions) Assume (B)(i), (L)-(i). Then, for every $t \in\left[s_{\lambda}, T\right]$,

$$
\mathbb{E}\left[\left|\tilde{x}_{1}(t)-\tilde{x}_{2}(t)\right|^{p}\right] \leq L_{\delta}\left(\left|s_{1}-s_{2}\right|^{p}+\left|x_{1}^{0}-x_{2}^{0}\right|^{p}\right) .
$$

for some constant $L_{\delta} \geq 0$ that depends only on $d, m, p, T, \delta, \nu(Z), C_{i}, L_{i}$, for $i=1, \ldots, 4$.

Lemma 2.6. (Moduli with a concavity property) Let $\xi$ be a nonnegative random variable on a probability space $(\Omega, \mathcal{F}, \mathbb{P})$ and let $\omega$ be a semiconcavity modulus. Then

$$
\mathbb{E}\left[\xi^{2} \omega^{2}(\xi)\right] \leq\left(\mathbb{E}\left[\xi^{r(2-\beta)}\right]\right)^{\frac{2}{r(2-\beta)}} \omega^{2}(\mathbb{E}[\xi])
$$


provided that $\gamma$, defined by setting $\gamma(\rho)=\left(\rho^{\beta} \omega^{2}(\rho)\right)^{q}$ for all $\rho \geq 0$, where $0 \leq \beta \leq 2,1 \leq r, q \leq \infty, r^{-1}+q^{-1}=1$, is concave.

Lemma 2.7. ( $C^{1, \omega}$-estimates in terms of initial conditions) Assume $(B)-(i)$, (L)-(i), (S)-(i) and (MP)-(i) or (MC)-(i). Then, for $\tilde{x}_{\lambda}(\cdot)=\lambda \tilde{x}_{1}(\cdot)+(1-$ $\lambda) \tilde{x}_{2}(\cdot)$, and for every $t \in\left[s_{\lambda}, T\right]$,

$$
\begin{aligned}
& \left(\mathbb{E}\left[\left|\tilde{x}_{\lambda}(t)-x_{\lambda}(t)\right|^{2}\right]\right)^{1 / 2} \\
& \quad \leq \lambda(1-\lambda)\left(\left|s_{1}-s_{2}\right|+\left|x_{1}^{0}-x_{2}^{0}\right|\right) \omega_{\delta}\left(\left|s_{1}-s_{2}\right|+\left|x_{1}^{0}-x_{2}^{0}\right|\right)
\end{aligned}
$$

where

$$
\omega_{\delta}(\rho)=\sum_{i=1}^{4} c_{i}^{\prime} \omega_{i}\left(c_{i} \rho\right)+c_{5} \rho, \quad \rho \geq 0,
$$

with constants $c_{i}, c_{i}^{\prime} \geq 0$ for $i=1,2,3,4, c_{5} \geq 0$ depending only on $d, m, p, T$, $\delta, C_{i}, L_{i}$, for $i=1, \ldots, 4$.

Lemma 2.8. Let $\xi$ be a nonnegative random variable on a probability space $(\Omega, \mathcal{F}, \mathbb{P})$ and $\omega$ a semiconcavity modulus. Then

$$
\mathbb{E}[\xi \omega(\xi)] \leq\left(\mathbb{E}\left[\xi^{r(1-\beta)}\right]\right)^{\frac{1}{r(1-\beta)}} \omega(\mathbb{E}[\xi])
$$

provided that $\gamma$, defined by setting $\gamma(\rho)=\left(\rho^{\beta} \omega(\rho)\right)^{q}$ for all $\rho \geq 0$, where $0 \leq \beta \leq 1,1 \leq r, q \leq \infty, q^{-1}+r^{-1}=1$, is concave.

Proof of Theorem 2.2. We have

$$
\begin{aligned}
& \lambda J_{R_{1}}\left(s_{1}, x_{1}^{0}, \alpha_{1}(\cdot)\right)+(1-\lambda) J_{R_{2}}\left(s_{2}, x_{2}^{0}, \alpha_{2}(\cdot)\right)-J_{R}\left(s_{\lambda}, x_{\lambda}^{0}, \alpha(\cdot)\right) \\
& =\lambda \mathbb{E}\left[\int_{s_{1}}^{T} L\left(t, x_{1}(t), \alpha_{1}(t)\right) d t\right]+(1-\lambda) \mathbb{E}\left[\int_{s_{2}}^{T} L\left(t, x_{2}(t), \alpha_{2}(t)\right) d t\right] \\
& \quad-\mathbb{E}\left[\int_{s_{\lambda}}^{T} L\left(t, x_{\lambda}(t), \alpha(t)\right) d t\right]+\lambda \mathbb{E}\left[\psi\left(x_{1}(T)\right)\right] \\
& \quad+(1-\lambda) \mathbb{E}\left[\psi\left(x_{2}(T)\right)\right]-\mathbb{E}\left[\psi\left(x_{\lambda}(T)\right)\right] .
\end{aligned}
$$

In the first two integrals we apply the change of variables $\tau_{1}, \tau_{2}$, respectively [defined as in (2.10)] and noticing that $\alpha_{i}(\cdot) \circ \tau_{i}^{-1}=\alpha(\cdot)$ for $i=1,2$, we find

$$
\begin{aligned}
\lambda J_{R_{1}}\left(s_{1}, x_{1}^{0}, \alpha_{1}(\cdot)\right)+(1-\lambda) J_{R_{2}}\left(s_{2}, x_{2}^{0}, \alpha_{2}(\cdot)\right)-J_{R}\left(s_{\lambda}, x_{\lambda}^{0}, \alpha(\cdot)\right) \\
=\int_{s_{\lambda}}^{T}\left(\frac{\lambda}{\dot{\tau}_{1}} \mathbb{E}\left[L\left(\tau_{1}^{-1}(t), \tilde{x}_{1}(t), \alpha(t)\right)\right]+\frac{1-\lambda}{\dot{\tau}_{2}} \mathbb{E}\left[L\left(\tau_{2}^{-1}(t), \tilde{x}_{2}(t), \alpha(t)\right)\right]\right. \\
\left.\quad-\mathbb{E}\left[L\left(t, x_{\lambda}(t), \alpha(t)\right)\right]\right) d t+\lambda \mathbb{E}\left[\psi\left(x_{1}(T)\right)\right] \\
\quad+(1-\lambda) \mathbb{E}\left[\psi\left(x_{2}(T)\right)\right]-\mathbb{E}\left[\psi\left(x_{\lambda}(T)\right)\right] \\
=\int_{s_{\lambda}}^{T} \mathbb{E}\left[\frac{\lambda}{\dot{\tau}_{1}} L\left(\tau_{1}^{-1}(t), \tilde{x}_{1}(t), \alpha(t)\right)\right.
\end{aligned}
$$




$$
\begin{aligned}
& \left.+\frac{1-\lambda}{\dot{\tau}_{2}} L\left(\tau_{2}^{-1}(t), \tilde{x}_{2}(t), \alpha(t)\right)-L\left(t, \tilde{x}_{\lambda}(t), \alpha(t)\right)\right] d t \\
& +\int_{s_{\lambda}}^{T} \mathbb{E}\left[L\left(t, \tilde{x}_{\lambda}(t), \alpha(t)\right)-L\left(t, x_{\lambda}(t), \alpha(t)\right)\right] d t \\
& +\mathbb{E}\left[\lambda \psi\left(x_{1}(T)\right)+(1-\lambda) \psi\left(x_{2}(T)\right)-\psi\left(\tilde{x}_{\lambda}(T)\right)\right] \\
& +\mathbb{E}\left[\psi\left(\tilde{x}_{\lambda}(T)\right)-\psi\left(x_{\lambda}(T)\right)\right],
\end{aligned}
$$

[recall that $\left.\tilde{x}_{\lambda}(\cdot)=\lambda \tilde{x}_{1}(\cdot)+(1-\lambda) \tilde{x}_{2}(\cdot)\right]$. Furthermore, noting that $\lambda / \dot{\tau}_{1}+$ $(1-\lambda) / \dot{\tau}_{2}=1$ as follows by differentiating (2.17), we get

$$
\begin{aligned}
\lambda J_{R_{1}} & \left(s_{1}, x_{1}^{0}, \alpha_{1}(\cdot)\right)+(1-\lambda) J_{R_{2}}\left(s_{2}, x_{2}^{0}, \alpha_{2}(\cdot)\right)-J_{R}\left(s_{\lambda}, x_{\lambda}^{0}, \alpha(\cdot)\right) \\
= & \int_{s_{\lambda}}^{T} \mathbb{E}\left[\lambda L\left(\tau_{1}^{-1}(t), \tilde{x}_{1}(t), \alpha(t)\right)+(1-\lambda) L\left(\tau_{2}^{-1}(t), \tilde{x}_{2}(t), \alpha(t)\right)\right. \\
& \left.-L\left(t, \tilde{x}_{\lambda}(t), \alpha(t)\right)\right] d t \\
& +\int_{s_{\lambda}}^{T}(1-\lambda)\left(\frac{1}{\dot{\tau}_{2}}-1\right) \mathbb{E}\left[L\left(\tau_{1}^{-1}(t), \tilde{x}_{1}(t), \alpha(t)\right)-L\left(\tau_{2}^{-1}(t), \tilde{x}_{2}(t), \alpha(t)\right)\right] d t \\
& +\int_{s_{\lambda}}^{T} \mathbb{E}\left[L\left(t, \tilde{x}_{\lambda}(t), \alpha(t)\right)-L\left(t, x_{\lambda}(t), \alpha(t)\right)\right] d t \\
& +\mathbb{E}\left[\lambda \psi\left(x_{1}(T)\right)+(1-\lambda) \psi\left(x_{2}(T)\right)-\psi\left(\tilde{x}_{\lambda}(T)\right)\right] \\
& +\mathbb{E}\left[\psi\left(\tilde{x}_{\lambda}(T)\right)-\psi\left(x_{\lambda}(T)\right)\right] .
\end{aligned}
$$

By (L)-(ii), (S)-(ii),

$$
\begin{aligned}
\lambda J_{R_{1}} & \left(s_{1}, x_{1}^{0}, \alpha_{1}(\cdot)\right)+(1-\lambda) J_{R_{2}}\left(s_{2}, x_{2}^{0}, \alpha_{2}(\cdot)\right)-J_{R}\left(s_{\lambda}, x_{\lambda}^{0}, \alpha(\cdot)\right) \\
\leq & \lambda(1-\lambda) \int_{s_{\lambda}}^{T} \mathbb{E}\left[\left(\left|\tau_{1}^{-1}(t)-\tau_{2}^{-1}(t)\right|+\left|\tilde{x}_{1}(t)-\tilde{x}_{2}(t)\right|\right)\right. \\
& \left.\times \omega_{5}\left(\left|\tau_{1}^{-1}(t)-\tau_{2}^{-1}(t)\right|+\left|\tilde{x}_{1}(t)-\tilde{x}_{2}(t)\right|\right)\right] d t \\
& +L_{5}(1-\lambda)\left(1-\frac{1}{\dot{\tau}_{2}}\right)\left(\left|\tau_{1}^{-1}(t)-\tau_{2}^{-1}(t)\right|+\mathbb{E}\left[\left|\tilde{x}_{1}(t)-\tilde{x}_{2}(t)\right|\right]\right) \\
& +L_{5} \int_{s_{\lambda}}^{T} \mathbb{E}\left[\left|\tilde{x}_{\lambda}(t)-x_{\lambda}(t)\right|\right] d t \\
& +\lambda(1-\lambda) \mathbb{E}\left[\left|\tilde{x}_{1}(T)-\tilde{x}_{2}(T)\right|\right] \omega_{6}\left(\mathbb{E}\left[\left|x_{1}(T)-x_{2}(T)\right|\right]\right) \\
& +L_{6} \mathbb{E}\left[\left|\tilde{x}_{\lambda}(T)-x_{\lambda}(T)\right|\right] .
\end{aligned}
$$

By Lemma 2.8 and Lemma 2.4, more specifically, (2.14), (2.18),

$$
\begin{aligned}
\lambda & J_{R_{1}}\left(s_{1}, x_{1}^{0}, \alpha_{1}(\cdot)\right)+(1-\lambda) J_{R_{2}}\left(s_{2}, x_{2}^{0}, \alpha_{2}(\cdot)\right)-J_{R}\left(s_{\lambda}, x_{\lambda}^{0}, \alpha(\cdot)\right) \\
\leq & C_{\delta} \lambda(1-\lambda)\left(\int _ { s _ { \lambda } } ^ { T } \mathbb { E } \left[\left(\left|s_{1}-s_{2}\right|\right.\right.\right. \\
& \left.\left.+\left|\tilde{x}_{1}(t)-\tilde{x}_{2}(t)\right|\right) \omega_{5}\left(C_{d}\left(\left|s_{1}-s_{2}\right|+\left|\tilde{x}_{1}(t)-\tilde{x}_{2}(t)\right|\right)\right)\right] d t \\
& +\mathbb{E}\left[\left|\tilde{x}_{1}(T)-\tilde{x}_{2}(T)\right| \omega_{6}\left(\left|\tilde{x}_{1}(T)-\tilde{x}_{2}(T)\right|\right)\right] \\
& \left.+\left(\left|s_{1}-s_{2}\right|+\left|x_{1}^{0}-x_{2}^{0}\right|\right)\right)+L_{5} \int_{s_{\lambda}}^{T} \mathbb{E}\left[\left|\tilde{x}_{\lambda}(t)-x_{\lambda}(t)\right|\right] d t
\end{aligned}
$$


for some constant $C_{\delta} \geq 0$ that depends only on $d, m, p, \delta, T, \nu(Z), C_{i}, L_{i}, i=$ $1, \ldots, 6$.

Under our assumptions on the moduli (MP) or (MC) Lemma 2.7 holds true, which, in case of assumptions (MC), we apply together with Lemma 2.8, in order to deduce, by using also a final time Lemma 2.5, that

$$
\begin{aligned}
& \lambda J_{R_{1}}\left(s_{1}, x_{1}^{0}, \alpha_{1}(\cdot)\right)+(1-\lambda) J_{R_{2}}\left(s_{2}, x_{2}^{0}, \alpha_{2}(\cdot)\right) \\
& \quad-J_{R}\left(s_{\lambda}, x_{\lambda}^{0}, \alpha(\cdot)\right) \leq \lambda(1-\lambda) \omega\left(\left|s_{1}-s_{2}\right|+\left|x_{1}^{0}-x_{2}^{0}\right|\right)+\left(\left|s_{1}-s_{2}\right|+\left|x_{1}^{0}-x_{2}^{0}\right|\right)
\end{aligned}
$$

for some $\omega$ as in (2.9). From this last estimate, since $R \in \mathcal{R}_{s_{\lambda}}$ and $\alpha(\cdot) \in$ $\mathcal{A}_{R}\left(s_{\lambda}, T\right)$ are arbitrary, it follows that $V$ is $\omega$-semiconcave.

Remark 2.9. Up to estimate (2.2) in the proof above we do not use the assumptions on the particular form of the moduli. This is important to notice for the general estimate (2.24) may by used to obtain generalized semiconcavity estimates for other types of moduli from those envisioned in Theorem 2.2.

It should be now rather straightforward to state results under the assumption that some of the moduli $\omega_{i}$ are of power type while the others satisfy suitable concavity properties (as stated in Lemmas 2.6 and 2.8).

In many cases of interest it is possible to choose the moduli $\omega_{i}$ concave, and by growth assumptions contained in (B), (L), it is also possible to assume these moduli $\omega_{i}$ bounded as well. This remark can be used to derive $\omega$-semiconcavity results by means of the following lemma.

Lemma 2.10. (bounded concave moduli) Fix $q, r \in[1, \infty]$ such that $1 / q+1 / r=$ 1 and let $\xi$ be as in Lemma 2.6 (or as in Lemma 2.8). Assume that $\omega^{\bar{q}}$ is concave for some $\bar{q}>0$, and $\omega$ is bounded by some constant $k \geq 0$. Then

$$
\mathbb{E}\left[\xi^{2} \omega^{2}(\xi)\right] \leq k^{1-\bar{q} /(2 q)}\left(\mathbb{E}\left[\xi^{2 r}\right]\right)^{1 / r} \omega^{\bar{q} /(2 q)}(\mathbb{E}[\xi])
$$

and

$$
\mathbb{E}[\xi \omega(\xi)] \leq k^{1-\bar{q} /(q)}\left(\mathbb{E}\left[\xi^{r}\right]\right)^{1 / r} \omega^{\bar{q} /(1 q)}(\mathbb{E}[\xi]) .
$$

Then this lemma can by used to prove the following theorem in the same fashion as we did with Theorem 2.2.

Theorem 2.11. (bounded concave moduli) Assume (B), (L), (SC). Assume in addition that, for suitable $1<q_{i}, r_{i}<\infty, \bar{q}_{i}>0$ such that $1 / q_{i}+1 / r_{i}=1$, for $i=1, \ldots, 6,2 r_{i} \leq p$ for $i=1, \ldots, 4, r_{5}, r_{6} \leq p$, maps $\omega_{i}^{\bar{q}_{i}}$ are concave and bounded. Then, for all $\delta \in] 0, T]$, the value function $V$ is $\omega$-semiconcave on $[0, T-\delta] \times \mathbb{R}^{d}$ for some modulus $\omega$ of the form

$$
\omega(\rho)=\sum_{i=1}^{4} c_{i}^{\prime} \omega_{i}^{\bar{q}_{i} /\left(2 q_{i}\right)}\left(c_{i} \rho\right)+\sum_{i=5}^{6} c_{i}^{\prime} \omega_{i}^{\bar{q}_{i} /\left(q_{i}\right)}\left(c_{i} \rho\right)+c_{7} \rho \quad \forall \rho \geq 0
$$

for constants $c_{i}, c_{i}^{\prime} \geq 0$ for $i=1, \ldots, 6, c_{7} \geq 0$ that depend only on $d, T, \delta, \nu(Z)$, $p, C_{i}, L_{i}, q_{i}, \overline{q_{i}}$ and upper bounds of $\omega_{i}$ for $i=1, \ldots, 6$.

Relying on the lemmas and techniques given above, one can obtain additional results on the time-space semiconcavity of the value function, estimating, 
if one so wishes, the semiconcavity modulus of the value function in terms of the moduli of the data (that is, results of the type of Theorems 2.2 and 2.11) when one assumes moduli of "mixed type", that is, some moduli of power type and the others having suitable concavity properties and/or being bounded. Since the resulting statements and method of proof of these results should be clear by now, we are not providing them here. We just emphasize that, in obtaining such results, the starting point is estimate (2.24), which holds true for any moduli $\omega_{i}, i=1, \ldots, 6$. Then one needs to apply Lemma 2.6 and/or the first part of Lemma 2.10, firstly, to obtain a new version of Lemma 2.7 (based on the assumptions on the type of the moduli $\left.o_{i}, i=1, \ldots, 4\right)$, and finally, one concludes by using this new version of Lemma 2.7, estimate (2.24) and/or Lemma 2.8 and/or the second part of Lemma 2.10 (whether and which of the said lemmas is to be used or not depends on the assumptions on $\omega_{i}$-s).

\section{Appendix}

Proof of Lemma 2.3. Fact 1. (Burkholder-Davis-Gundy inequalities [43]) For any $2 \leq p<\infty$ there exist $c_{p}^{\prime}, c_{p}>0$ such that

$$
\mathbb{E}\left[\left|\int_{s}^{t} \sigma(r) d W(r)\right|^{p}\right] \leq c_{p}^{\prime} \mathbb{E}\left[\left(\int_{s}^{t}|\sigma(r)|^{2} d r\right)^{p / 2}\right]
$$

and

$$
\begin{aligned}
\mathbb{E}\left[\left|\int_{s}^{t} \int_{F} H(r, z) \tilde{N}(d r d z)\right|^{p}\right] \leq & c_{p} \mathbb{E}\left[\int_{s}^{t} \int_{F}|H(r, z)|^{p} d r \nu(d z)\right] \\
+c_{p}^{\prime \prime} \mathbb{E} & {\left[\left(\int_{s}^{t} \int_{F}|H(r, z)|^{2} d r \nu(d z)\right)^{p / 2}\right] }
\end{aligned}
$$

for all for all predictable processes $\sigma \in L^{p}\left([s, T] \times \Omega, d t \otimes \mathbb{P} ; \mathbb{R}^{d \times m}\right), H \in$ $L^{p}\left([s, T] \times F \times \Omega, d t \otimes \nu \otimes \mathbb{P} ; \mathbb{R}^{d}\right)$, where $F$ is any measurable subset of $Z$; see, e.g., [1, Theroem 4.4.22, p. 263 and Theorem 4.4.23, p. 265 ], or [43, Section 2.5]. Actually, for $p=2$, by the $L^{2}$-isometry of stochastic integrals, we can take $c_{p}=c_{p}^{\prime}=1$ and $c_{p}^{\prime \prime}=0$ above, and these inequalities are in fact equalities.

By first compensating, that is, using $N=\tilde{N}+d t \nu(d z)$, and then inequality (3.2) with $F=E^{c}$, and Hölder's inequality, we obtain

$$
\mathbb{E}\left[\left|\int_{s}^{t} \int_{E^{c}} K(r, z) N(d r d z)\right|^{p}\right] \leq c \mathbb{E}\left[\int_{s}^{t} \int_{|z|>\delta}|K(r, z)|^{p} d r \nu(d z)\right]
$$

for come $c>0$ that depends only $p, T, \nu\left(E^{c}\right)$; recall that $\nu\left(E^{c}\right)<\infty$, a fact which is used above. Clearly (3.1) implies

$$
\mathbb{E}\left[\left|\int_{s}^{t} \sigma(r) d W(r)\right|^{p}\right] \leq c \mathbb{E}\left[\int_{s}^{t}|\sigma(r)|^{p} d r\right]
$$


and since $\nu$ is a finite measure, (3.2) implies

$$
\mathbb{E}\left[\left|\int_{s}^{t} \int_{F} H(r, z) \tilde{N}(d r d z)\right|^{p}\right] \leq c \mathbb{E}\left[\int_{s}^{t} \int_{F}|H(r, z)|^{p} d r \nu(d z)\right]
$$

for some constant $c \geq 0$ depending only on $p, T, \nu(Z), d$.

Fact 2. If, for $i=1,2$, we define

$$
\begin{aligned}
\mathbb{F}_{i} & =\mathbb{F} \circ \tau_{i}^{-1}=\left\{\mathcal{F}_{\tau_{i}^{-1}\left(t^{\prime}\right)}\right\}_{s_{\lambda} \leq t^{\prime} \leq T} \\
\tau_{i}(W)(t) & =\frac{1}{\sqrt{\tau_{i}}}\left(W\left(\tau_{i}(t)\right)-W\left(s_{\lambda}\right)\right), \quad s_{i} \leq t \leq T,
\end{aligned}
$$

then $\tau_{i}(W)$ is a $\left(\mathbb{R}^{m}\right.$-valued) Brownian motion on $\left(\Omega, \mathcal{F}, \mathbb{F} \circ \tau_{i}^{-1}, \mathbb{P}\right)$. Moreover, we have

$$
\int_{s_{i}}^{\tau_{i}^{-1}(t)} \sigma(r) \tau_{i}(W)(d r)=\int_{s_{\lambda}}^{t} \frac{1}{\sqrt{\dot{\tau}_{i}}} \sigma\left(\tau_{i}^{-1}\left(r^{\prime}\right)\right) W\left(d r^{\prime}\right)
$$

for all predictable processes $\sigma \in L^{2}\left(\left[s_{i}, \tau_{i}^{-1}(t)\right] \times \Omega, d r \otimes \mathbb{P} ; \mathbb{R}^{m \times d}\right), t \in\left[s_{i}, T\right]$.

Next, we use a transformation of a Poisson random measure with respect to affine time changes which is called Kulik's transformation. The reader interested for more information on this transformation is referred to papers [41,42], or even [33] for a quick and very readable introduction. We define

$$
\tau_{i}(N)\left(\left[s_{i}, t\right] \times \Delta\right)=N\left(\left[s_{\lambda}, \tau_{i}(t)\right] \times \Delta\right), \quad s_{i} \leq t \leq T, \Delta \in \mathcal{Z} .
$$

Fact 3. For each $i=1,2, \tau_{i}(N)$ is a Poisson random measure on the filtered probability space $\left(\Omega, \mathcal{F}, \mathbb{F} \circ \tau_{i}^{-1}, \mathbb{Q}_{i}\right)$, where $\mathbb{Q}_{i}$ is another probability on $(\Omega, \mathcal{F})$ which is absolutely continuous with respect to $\mathbb{P}$ and has Radon-Nikodym density

$$
\frac{d \mathbb{Q}_{i}}{d \mathbb{P}}=\exp \left\{-\ln \left(\frac{T-s_{\lambda}}{T-s_{i}}\right) \tau_{i}(N)\left(\left[s_{i}, T\right] \times Z\right)+\left(s_{i}-s_{\lambda}\right) \nu(Z)\right\},
$$

while the time changed filtration $\mathbb{F} \circ \tau_{i}^{-1}$ is defined in (3.6). Moreover, we have the following change of variables formulas:

$$
\begin{aligned}
& \int_{s_{i}}^{\tau_{i}^{-1}(t)} \int_{E} H(r, z) \widetilde{\tau_{i}(N)}(d r d z)^{\mathbb{Q}_{i}} \\
& =\int_{s_{\lambda}}^{t} \int_{E} H\left(\tau_{i}^{-1}\left(r^{\prime}\right), z\right) \widetilde{N}\left(d r^{\prime} d z\right)^{\mathbb{P}} \\
& \quad+\left(1-\frac{1}{\dot{\tau}_{i}}\right) \int_{s_{\lambda}}^{t} \int_{E} H\left(\tau_{i}^{-1}\left(r^{\prime}\right), z\right) d r^{\prime} \nu(d z) ; \\
& \int_{s_{i}}^{\tau_{i}^{-1}(t)} \int_{E^{c}} K(r, z) \tau_{i}(N)(d r d z)^{\mathbb{Q}_{i}} \\
& =\int_{s_{\lambda}}^{t} \int_{E^{c}} K\left(\tau_{i}^{-1}\left(r^{\prime}\right), z\right) N\left(d r^{\prime} d z\right)^{\mathbb{P}}
\end{aligned}
$$


for all predictable processes $H \in L^{2}\left(\left[s_{i}, \tau_{i}^{-1}(t)\right] \times E \times \Omega, d r \otimes \nu \otimes \mathbb{Q}_{i} ; \mathbb{R}^{d}\right)$, and $K \in L^{2}\left(\left[s_{i}, \tau_{i}^{-1}(t)\right] \times E^{c} \times \Omega, d t \otimes \nu \otimes \mathbb{P} ; \mathbb{R}^{d}\right), t \in\left[s_{i}, T\right]$. We have added the symbols of probability measures $\mathbb{Q}_{i}$ or $\mathbb{P}$ to the integrals above in order to emphasize the probability space on which each integral is defined.

We have, by (3.7), (3.10), (3.11),

$$
\begin{aligned}
\tilde{x}_{i}(t)-x_{i}^{0}= & \int_{s_{\lambda}}^{t} \frac{1}{\dot{\tau}_{i}} b\left(\tau_{i}^{-1}\left(r^{\prime}\right), \tilde{x}_{i}\left(r^{\prime}\right), \alpha\left(r^{\prime}\right)\right) d r^{\prime} \\
& +\int_{s_{\lambda}}^{t} \frac{1}{\sqrt{\dot{\tau}_{i}}} \sigma\left(\tau_{i}^{-1}\left(r^{\prime}\right), \tilde{x}_{i}\left(r^{\prime}\right), \alpha\left(r^{\prime}\right)\right) W(d r)^{\mathbb{P}} \\
& +\int_{s_{\lambda}}^{t} \int_{E} H\left(\tau_{i}^{-1}\left(r^{\prime}\right), \tilde{x}_{i}\left(r^{\prime}\right), z, \alpha\left(r^{\prime}\right)\right) \widetilde{\tau_{i}(N)}\left(d r^{\prime} d z\right)^{\mathbb{P}} \\
& +\left(1-\frac{1}{\dot{\tau}_{i}}\right) \int_{s_{\lambda}}^{t} \int_{E} H\left(\tau_{i}^{-1}\left(r^{\prime}\right), \tilde{x}_{i}\left(r^{\prime}\right), z, \alpha\left(r^{\prime}\right)\right) d r^{\prime} \nu(d z) \\
& +\int_{s_{\lambda}}^{t} \int_{E^{c}} K\left(\tau_{i}^{-1}\left(r^{\prime}\right), \tilde{x}_{i}\left(r^{\prime}\right), z, \alpha\left(r^{\prime}\right)\right) \tau_{i}(N)\left(d r^{\prime} d z\right)^{\mathbb{P}} .
\end{aligned}
$$

We have also used the fact that $\tau_{i}(W)$ is also a Brownian motion with respect to probability $\mathbb{Q}_{i}$ and

$$
\begin{gathered}
\int_{s_{i}}^{\tau_{i}^{-1}(t)} \sigma\left(\tau_{i}^{-1}(r), \tilde{x}_{i}(r)\right)^{\mathbb{Q}_{i}} \tau_{i}(W)(d r) \\
=\int_{s_{i}}^{\tau_{i}^{-1}(t)} \sigma\left(\tau_{i}^{-1}(r), \tilde{x}_{i}(r)\right)^{\mathbb{P}} \tau_{i}(W)(d r)
\end{gathered}
$$

because $\mathbb{Q}_{i}$ is absolutely continuous with respect to $\mathbb{P}$ and $d \mathbb{Q}_{i} / d \mathbb{P}$ is bounded almost surely with respect to $\mathbb{P}$ (and hence $\mathbb{Q}_{i}$ ) and simple change of variable formula for ordinary (deterministic) integrals:

$$
\int_{s_{i}}^{\tau_{i}^{-1}(t)} b(r, x(r)) d r=\int_{s_{\lambda}}^{t} \frac{1}{\dot{\tau}_{i}} b\left(\tau_{i}^{-1}\left(r^{\prime}\right), \tilde{x}_{i}\left(r^{\prime}\right)\right) d r^{\prime} .
$$

Subtracting the two identities in (3.12) for $i=1,2$, taking the expected value of the $p$-th moment of the resulting difference and using moment inequalities (3.4), (3.5), we obtain (2.12). The other estimate (2.13) is proved similarly.

Proof of Lemma 2.5. For notational brevity, let $f_{1}=b, f_{2}=\sigma$,

$$
\begin{aligned}
& f_{3}:[0, T] \times \mathbb{R}^{d} \times A \rightarrow L^{p}\left(E, \nu ; \mathbb{R}^{d}\right), \\
& {[0, T] \times \mathbb{R}^{d} \times A \ni(t, x, \alpha) \mapsto H(t, x, \cdot, \alpha) \in L^{p}\left(E, \nu ; \mathbb{R}^{)}\right.}
\end{aligned}
$$

and

$$
\begin{aligned}
& f_{4}:[0, T] \times \mathbb{R}^{d} \times A \rightarrow L^{p}\left(E, \nu ; \mathbb{R}^{d}\right), \\
& {[0, T] \times \mathbb{R}^{d} \times A \ni(t, x, \alpha) \mapsto K(t, x, \cdot, \alpha) \in L^{p}\left(E^{c}, \nu ; \mathbb{R}^{d}\right) ;}
\end{aligned}
$$


let also $\phi_{1}(\dot{\tau})=1 / \dot{\tau}, \phi_{2}(\dot{\tau})=1 / \sqrt{\dot{\tau}}, \phi_{3}(\dot{\tau})=1, \phi_{4}(\dot{\tau})=1-1 / \dot{\tau}$ for all $\dot{\tau} \in \mathbb{R}$, $i=1, \ldots, 4$.

Since $^{5}$

$$
\begin{aligned}
(\mathbb{E} & {\left.\left[\left\|\phi_{i}\left(\dot{\tau}_{1}\right) f_{i}\left(\tau_{1}^{-1}(r), \tilde{x}_{1}(r)\right)-\phi_{i}\left(\dot{\tau}_{2}\right) f_{i}\left(\tau_{2}^{-1}(r), \tilde{x}_{2}(r)\right)\right\|^{p}\right]\right)^{1 / p} } \\
\leq & \left|\phi_{i}\left(\dot{\tau}_{1}\right)-\phi_{i}\left(\dot{\tau}_{2}\right)\right|\left(\mathbb{E}\left[\left\|f_{i}\left(\tau_{1}^{-1}(r), \tilde{x}_{1}(r)\right)\right\|^{p}\right]\right)^{1 / p} \\
& +\phi_{i}\left(\dot{\tau}_{2}\right)\left(\mathbb{E}\left[\left\|f_{i}\left(\tau_{1}^{-1}(r), \tilde{x}_{1}(r)\right)-f_{i}\left(\tau_{2}^{-1}(r), \tilde{x}_{1}(r)\right)\right\|^{p}\right]\right)^{1 / p}
\end{aligned}
$$

for $i=1,2,3,4$, then, by Lemma 2.4, inequalities (3.4), (3.5), (3.3) and assumption (B)-(i), we deduce

$$
\begin{aligned}
& \left(\mathbb{E}\left[\left\|\phi_{i}\left(\dot{\tau}_{1}\right) f_{i}\left(\tau_{1}^{-1}(r), \tilde{x}_{1}(r)\right)-\phi_{i}\left(\dot{\tau}_{2}\right) f_{i}\left(\tau_{1}^{-1}(r), \tilde{x}_{1}(r)\right)\right\|^{p}\right]\right)^{1 / p} \\
& \quad \leq C_{\delta}\left(\left|s_{1}-s_{2}\right|+\left(\mathbb{E}\left[\left\|f_{i}\left(\tau_{1}^{-1}(r), \tilde{x}_{1}(r)\right)-f_{i}\left(\tau_{2}^{-1}(r), \tilde{x}_{1}(r)\right)\right\|^{p}\right]\right)^{1 / p}\right)
\end{aligned}
$$

for some constant $C_{\delta} \geq 0$ that depends on $d, m, p, \delta, T, \nu(Z), C_{i}, i=1, \ldots, 4$. This in turn, by Lemma 2.3, more specifically, by (2.12), and assumption (L)(i) yields

$\mathbb{E}\left[\left|\tilde{x}_{1}(t)-\tilde{x}_{2}(t)\right|^{p}\right] \leq C_{\delta}\left(\left|s_{1}-s_{2}\right|^{p}+\left|x_{1}^{0}-x_{2}^{0}\right|^{p}+\int_{s_{\lambda}}^{t} \mathbb{E}\left[\left|\tilde{x}_{1}(t)-\tilde{x}_{2}(t)\right|^{p}\right] d r\right)$

for another constant $C_{\delta} \geq 0$ that depends on $m, d, \delta, p, T, \nu(Z), C_{i}, L_{i}, i=$ $1, \ldots, 4$. This last estimate yields the claimed estimate (2.19) via Gronwall's inequality.

Proof of Lemma 2.6. By Hölder's and Jensen's inequalities we can estimate as follows

$$
\begin{aligned}
\mathbb{E}\left[\xi^{2} \omega(\xi)^{2}\right] & =\mathbb{E}\left[\left(\xi^{2-\beta}\right)\left(\xi^{\beta} \omega(\xi)^{2}\right)\right] \\
& \leq\left(\mathbb{E}\left[\xi^{r(2-\beta)}\right]\right)^{\frac{1}{r}}(\mathbb{E}[\gamma(\xi)])^{\frac{1}{q}} \\
& \leq\left(\mathbb{E}\left[\xi^{r(2-\beta)}\right]\right)^{\frac{1}{r}}(\gamma(\mathbb{E}[\xi]))^{\frac{1}{q}} \\
& =\left(\mathbb{E}\left[\xi^{r(2-\beta)}\right]\right)^{\frac{1}{r}}(\mathbb{E}[\xi])^{\beta} \omega^{2}(\mathbb{E}[\xi])
\end{aligned}
$$

from which, by a further application of Hölder's inequality, follows (2.20).

Lemmas 2.8 and 2.10 are proved similarly.

\footnotetext{
${ }^{5}$ Here the norms $\left\|f_{i}\right\|$ stand for the Euclidean norm on $\mathbb{R}^{d}$, for $i=1,2$, the standard norm of $L^{p}\left(E, \nu ; \mathbb{R}^{d}\right)$ for $i=3$ and the standard norm of $L^{p}\left(E^{c}, \nu ; \mathbb{R}^{d}\right)$ for for $i=4$.
} 
Proof of Lemma 2.7. We can write

$$
\begin{aligned}
& \lambda \phi_{i}\left(\dot{\tau}_{1}\right) f_{i}\left(\tau_{1}^{-1}(r), \tilde{x}_{1}(r)\right)+(1-\lambda) \phi_{i}\left(\dot{\tau}_{2}\right) f_{i}\left(\tau_{2}^{-1}(r), \tilde{x}_{2}(r)\right)-f\left(r, x_{\lambda}(r)\right) \\
&= \lambda f_{i}\left(\tau_{1}^{-1}(r), \tilde{x}_{1}(r)\right)+(1-\lambda) f_{i}\left(\tau_{2}^{-1}(r), \tilde{x}_{2}(r)\right)-f_{i}\left(r, \tilde{x}_{\lambda}(r)\right) \\
&+\left(\lambda \phi_{i}\left(\dot{\tau}_{1}\right)+(1-\lambda) \phi_{i}\left(\dot{\tau}_{2}\right)-1\right) f_{i}\left(\tau_{1}^{-1}(r), \tilde{x}_{1}(r)\right) \\
&+(1-\lambda)\left(1-\phi_{i}\left(\dot{\tau}_{2}\right)\right)\left(f_{i}\left(\tau_{1}^{-1}(r), \tilde{x}_{1}(r)\right)-f_{i}\left(\tau_{2}^{-1}(r), \tilde{x}_{2}(r)\right)\right) \\
&+f_{i}\left(r, \tilde{x}_{\lambda}(r)\right)-f_{i}\left(r, x_{\lambda}(r)\right)
\end{aligned}
$$

$i=1, \ldots, 4$, where maps $f_{i}, \phi_{i}$ are defined as in the proof of Lemma 2.3 above. By Lemma 2.4 we deduce that

$$
\left|\lambda \phi_{i}\left(\dot{\tau}_{1}\right)+(1-\lambda) \phi_{i}\left(\dot{\tau}_{2}\right)-1\right| \leq \frac{1}{\delta^{2}} \lambda(1-\lambda)\left|s_{1}-s_{2}\right|^{2}
$$

for $i=1, \ldots, 4$. Indeed, for $i=3$ the left-hand side of (3.14) obviously vanishes; by differentiating (2.17) one discovers that it also vanishes for $i=1$; for $i=4$ it vanishes too as follows from the case for $i=1$, and the case $i=2$ is covered by $(2.16)$.

Using identity (3.13), inequalities (3.1), (3.2), (3.3) and assumptions (B)(i), (L)-(i), (S)-(i), we obtain,

$$
\begin{aligned}
\mathbb{E}[\| & \left.\lambda \phi_{i}\left(\dot{\tau}_{1}\right) f_{i}\left(\tau_{1}^{-1}(r), \tilde{x}_{1}(r)\right)+(1-\lambda) \phi_{i}\left(\dot{\tau}_{2}\right) f_{i}\left(\tau_{2}^{-1}(r), \tilde{x}_{2}(r)\right)-\left.f\left(r, x_{\lambda}(r)\right)\right|^{2}\right] \\
\leq & (\lambda(1-\lambda))^{2} \mathbb{E}\left[\left(\left(\left|\tau_{1}^{-1}(r)-\tau_{2}^{-1}(r)\right|^{2}+\left|\tilde{x}_{1}(r)-\tilde{x}_{2}(r)\right|^{2}\right)\right.\right. \\
& \left.\times\left(\omega_{i}\left(\left|\tau_{1}^{-1}(r)-\tau_{2}^{-1}(r)\right|+\mid \tilde{x}_{1}(r)-\tilde{x}_{2}(r)\right)\right)^{2}\right] \\
& \left.+C_{\delta}\left|s_{1}-s_{2}\right|^{2}\left(\left|s_{1}-s_{2}\right|^{2}+\mathbb{E}\left[\left|\tilde{x}_{1}(r)-\tilde{x}_{2}(r)\right|^{2}\right]\right)\right)+C_{\delta} \mathbb{E}\left[\left|\tilde{x}_{\lambda}(r)-x_{\lambda}(r)\right|^{2}\right]
\end{aligned}
$$

for some constant $C_{\delta} \geq 0$ that depend on $\delta, T, C_{i}, L_{i}, i=1, \ldots, 4$. Hence, by Lemma 2.19,

$$
\begin{aligned}
& \mathbb{E}\left[\left\|\lambda \phi_{i}\left(\dot{\tau}_{1}\right) f_{i}\left(\tau_{1}^{-1}(r), \tilde{x}_{1}(r)\right)+(1-\lambda) \phi_{i}\left(\dot{\tau}_{2}\right) f_{i}\left(\tau_{2}^{-1}(r), \tilde{x}_{2}(r)\right)-f\left(r, x_{\lambda}(r)\right)\right\|^{2}\right] \\
& \leq C_{\delta}(\lambda(1-\lambda))^{2} \mathbb{E}\left[\left(\left|s_{1}-s_{2}\right|+\left|\tilde{x}_{1}(r)-\tilde{x}_{2}(r)\right|\right)^{2}\right. \\
& \left.\times\left(\omega_{i}\left(C_{\varepsilon}\left(\left|s_{1}-s_{2}\right|+\left|\tilde{x}_{1}(r)-\tilde{x}_{2}(r)\right|\right)\right)\right)^{2}\right] \\
& \quad+C_{\delta}\left|s_{1}-s_{2}\right|^{2}\left(\left|s_{1}-s_{2}\right|^{2}+\left|x_{1}^{0}-x_{2}^{0}\right|^{2}\right)+C_{\delta} \mathbb{E}\left[\left|\tilde{x}_{\lambda}(r)-x_{\lambda}(r)\right|^{2}\right] .
\end{aligned}
$$

Thus, by Lemma 2.3, and specifically, estimate (2.13),

$$
\begin{aligned}
\mathbb{E} & {\left[\left|\tilde{x}_{\lambda}(t)-x_{\lambda}(t)\right|^{2}\right] \leq C_{\delta}(\lambda(1-\lambda))^{2} \sum_{i=1}^{4} \int_{s_{\lambda}}^{t} \mathbb{E}\left[\left(\left|s_{1}-s_{2}\right|+\left|\tilde{x}_{1}(r)-\tilde{x}_{2}(r)\right|\right)^{2}\right.} \\
& \left.\times\left(\omega_{i}\left(C_{\varepsilon}\left(\left|s_{1}-s_{2}\right|+\left|\tilde{x}_{1}(r)-\tilde{x}_{2}(r)\right|\right)\right)\right)^{2}\right] d r \\
& +C_{\delta}\left|s_{1}-s_{2}\right|^{2}\left(\left|s_{1}-s_{2}\right|^{2}+\left|x_{1}^{0}-x_{2}^{0}\right|^{2}\right) \\
& +C_{\delta} \int_{s_{\lambda}}^{t} \mathbb{E}\left[\left|\tilde{x}_{\lambda}(r)-x_{\lambda}(r)\right|^{2}\right] d r,
\end{aligned}
$$

for some $C_{\delta} \geq 0$ depending only on $d, m, T, \delta, \nu(Z), C_{i}, L_{i}, i=1, \ldots, 4$. 
Up to this point our estimates hold true for any moduli $\omega_{i}$-s; now we have to distinguish the two cases. Let us first treat the case (MP)-(i). In this case we have

$$
\begin{aligned}
\mathbb{E}\left[\left|\tilde{x}_{\lambda}(t)-x_{\lambda}(t)\right|^{2}\right] \leq & C_{\delta}(\lambda(1-\lambda))^{2} \sum_{i=1}^{4} \int_{s_{\lambda}}^{t}\left(\mathbb { E } \left[k _ { i } ^ { 2 } \left(\left|s_{1}-s_{2}\right|\right.\right.\right. \\
& \left.\left.\left.+\left|\tilde{x}_{1}(r)-\tilde{x}_{2}(r)\right|\right)^{2+2 \alpha_{i}}\right]\right) d r \\
& +C_{\delta}\left|s_{1}-s_{2}\right|^{2}\left(\left|s_{1}-s_{2}\right|^{2}+\left|x_{1}^{0}-x_{2}^{0}\right|^{2}\right) \\
& +C_{\delta} \int_{s_{\lambda}}^{t} \mathbb{E}\left[\left|\tilde{x}_{\lambda}(r)-x_{\lambda}(r)\right|^{2}\right] d r
\end{aligned}
$$

for some $C_{\delta} \geq 0$ depending only on $d, m, T, \delta, \nu(Z), C_{i}, L_{i}, i=1, \ldots, 4$. Then, by Lemma 2.5 again, taking into account that $p \geq 2+2 \alpha_{i}$ for $i=1, \ldots, 4$, we get

$$
\begin{aligned}
\mathbb{E}\left[\left|\tilde{x}_{\lambda}(t)-x_{\lambda}(t)\right|^{2}\right] \leq & C_{\delta}(\lambda(1-\lambda))^{2} \sum_{i=1}^{4} k_{i}^{2}\left(\left|s_{1}-s_{2}\right|+\left|x_{1}^{0}-x_{2}^{0}\right|\right)^{2+2 \alpha_{i}} \\
& +C_{\delta}\left|s_{1}-s_{2}\right|^{2}\left(\left|s_{1}-s_{2}\right|^{2}+\left|x_{1}^{0}-x_{2}^{0}\right|^{2}\right) \\
& +C_{\delta} \int_{s_{\lambda}}^{t} \mathbb{E}\left[\left|\tilde{x}_{\lambda}(r)-x_{\lambda}(r)\right|^{2}\right] d r
\end{aligned}
$$

for some $C_{\delta} \geq 0$ depending only on $d, m, T, \delta, \nu(Z), C_{i}, L_{i}, i=1, \ldots, 4$. By Gronwall's inequality and since $p \geq 2+2 \alpha_{i}$ for $i=1, \ldots, 4$, we derive the claimed estimate (2.21) with $\omega_{\delta}$ as in (2.22).

Next let us consider the case (MC)-(i). By (3.15) and Lemma 2.6, we obtain

$$
\begin{aligned}
& \mathbb{E}\left[\left|\tilde{x}_{\lambda}(t)-x_{\lambda}(t)\right|^{2}\right] \leq C_{\delta}(\lambda(1-\lambda))^{2} \sum_{i=1}^{4} \int_{s_{\lambda}}^{t}\left(\mathbb { E } \left[\left(\left|s_{1}-s_{2}\right|\right.\right.\right. \\
& \left.\left.\left.\quad+\left|\tilde{x}_{1}(r)-\tilde{x}_{2}(r)\right|\right)^{r_{i}\left(2-\beta_{i}\right)}\right]\right)^{\frac{2}{r_{i}\left(2-\beta_{i}\right)}} \\
& \quad \times\left(\omega_{i}\left(\mathbb{E}\left[C_{\delta}\left(\left|s_{1}-s_{2}\right|+\left|\tilde{x}_{1}(r)-\tilde{x}_{2}(r)\right|\right)\right]\right)\right)^{2} d r \\
& \quad+C_{\delta}\left|s_{1}-s_{2}\right|^{2}\left(\left|s_{1}-s_{2}\right|^{2}+\left|x_{1}^{0}-x_{2}^{0}\right|^{2}\right)+C_{\delta} \int_{s_{\lambda}}^{t} \mathbb{E}\left[\left|\tilde{x}_{\lambda}(r)-x_{\lambda}(r)\right|^{2}\right] d r
\end{aligned}
$$

for some $C_{\delta} \geq 0$ depending only on $d, m, T, \delta, \nu(Z), C_{i}, L_{i}, i=1, \ldots, 4$. But by assumption (MC), $p \geq r_{i}\left(2-\beta_{i}\right)$, so that by Lemma 2.5 we obtain

$$
\begin{aligned}
& \mathbb{E}\left[\left|\tilde{x}_{\lambda}(t)-x_{\lambda}(t)\right|^{2}\right] \leq C_{\delta}(\lambda(1-\lambda))^{2} \sum_{i=1}^{4}\left(\left(\left|s_{1}-s_{2}\right|+\left|x_{1}^{0}-x_{2}^{0}\right|\right)\right. \\
& \left.\quad \times \omega_{i}\left(C_{\delta}\left(\left|s_{1}-s_{2}\right|+\left|x_{1}^{0}-x_{2}^{0}\right|\right)\right)\right)^{2} \\
& \quad+C_{\delta}\left|s_{1}-s_{2}\right|^{2}\left(\left|s_{1}-s_{2}\right|^{2}+\left|x_{1}^{0}-x_{2}^{0}\right|^{2}\right)+C_{\delta} \int_{s_{\lambda}}^{t} \mathbb{E}\left[\left|\tilde{x}_{\lambda}(r)-x_{\lambda}(r)\right|^{2}\right] d r
\end{aligned}
$$


for some $C_{\delta} \geq 0$ depending only on $p, d, m, T, \delta, \nu(Z), C_{i}, L_{i}, i=1, \ldots, 4$. Finally, an application of Gronwall's inequality yields the estimate (2.21) with $\omega_{\delta}$ as in $(2.22)$.

\section{Acknowledgements}

I am much in debt to and thank an anonymous reviewer, whose extensive comments, corrections and suggestions helped me very much in improve the paper. I would like to thank also Prof. Piermarco Cannarsa and Prof. Martino Bardi for useful conversations and their advise.

Open Access. This article is distributed under the terms of the Creative Commons Attribution 4.0 International License (http://creativecommons.org/licenses/ by/4.0/), which permits unrestricted use, distribution, and reproduction in any medium, provided you give appropriate credit to the original author(s) and the source, provide a link to the Creative Commons license, and indicate if changes were made.

Publisher's Note Springer Nature remains neutral with regard to jurisdictional claims in published maps and institutional affiliations.

\section{References}

[1] Applebaum, D.: Lévy Processes and Stochastic Calculus. Cambridge Studies in Advanced Mathematics, vol. 116, 2nd edn. Cambridge University Press, Cambridge (2009)

[2] Bardi, M., Capuzzo-Dolcetta, I.: Optimal Control and Viscosity Solutions of Hamilton-Jacobi-Bellman Equations. Birkhäuser, Basel (2008). (reprint of the 1997 original edition)

[3] Bardi, Martino, Feleqi, Ermal: Nonlinear elliptic systems and mean-field games. NoDEA Nonlinear Differ. Equ. Appl. 23(4), 32 (2016)

[4] Barles, Guy, Chasseigne, Emmanuel, Ciomaga, Adina, Imbert, Cyril: Lipschitz regularity of solutions for mixed integro-differential equations. J. Differ. Equ. 252(11), 6012-6060 (2012)

[5] Barles, Guy, Chasseigne, Emmanuel, Imbert, Cyril: Hölder continuity of solutions of second-order non-linear elliptic integro-differential equations. J. Eur. Math. Soc. 13(1), 1-26 (2011)

[6] Barles, Guy, Imbert, Cyril: Second-order elliptic integro-differential equations: viscosity solutions' theory revisited. Ann. Inst. H. Poincaré Anal. Non Linéaire 25(3), 567-585 (2008)

[7] Bensoussan, A., Lions, J.-L.: Impulse Control and Quasivariational Inequalities $\mu$. Gauthier-Villars, Montrouge (1984). (translated from the French by J. M. Cole) 
[8] Benton, Stanley H: The Hamilton-Jacobi Equation: A Global Approach. Elsevier, Amsterdam (1977)

[9] Bian, Baojun, Guan, Pengfei: Convexity preserving for fully nonlinear parabolic integro-differential equations. Methods Appl. Anal. 15(1), 39-51 (2008)

[10] Brasco, Lorenzo, Lindgren, Erik: Higher sobolev regularity for the fractional p-laplace equation in the superquadratic case. Adv. Math. 304, 300-354 (2017)

[11] Buckdahn, Rainer, Cannarsa, Piermarco, Quincampoix, Marc: Lipschitz continuity and semiconcavity properties of the value function of a stochastic control problem. NoDEA Nonlinear Differ. Equ. Appl. 17(6), 715-728 (2010)

[12] Buckdahn, Rainer, Huang, Jianhui, Li, Juan: Regularity properties for general HJB equations: a backward stochastic differential equation method. SIAM J. Control Optim. 50(3), 1466-1501 (2012)

[13] Caffarelli, Luis, Chan, Chi Hin, Vasseur, Alexis: Regularity theory for parabolic nonlinear integral operators. J. Am. Math. Soc 24(3), 849-869 (2011)

[14] Caffarelli, Luis, Silvestre, Luis: Regularity theory for fully nonlinear integrodifferential equations. Commun. Pure Appl. Math. 62(5), 597-638 (2009)

[15] Caffarelli, Luis, Silvestre, Luis: The Evans-Krylov theorem for nonlocal fully nonlinear equations. Ann. Math. (2) 174(2), 1163-1187 (2011)

[16] Caffarelli, Luis, Silvestre, Luis: Regularity results for nonlocal equations by approximation. Arch. Ration. Mech. Anal. 200(1), 59-88 (2011)

[17] Caffarelli, Luis A., Vasseur, Alexis: Drift diffusion equations with fractional diffusion and the quasi-geostrophic equation. Ann. Math. (2) 171(3), 1903-1930 (2010)

[18] Caffarelli, Luis A., Vasseur, Alexis F.: The De Giorgi method for regularity of solutions of elliptic equations and its applications to fluid dynamics. Discrete Contin. Dyn. Syst. Ser. S 3(3), 409-427 (2010)

[19] Cannarsa, Piermarco, Frankowska, Halina: Some characterizations of optimal trajectories in control theory. SIAM J. Control Optim. 29(6), 1322-1347 (1991)

[20] Cannarsa, Piermarco, Sinestrari, Carlo: Convexity properties of the minimum time function. Calc. Var. Partial Differ. Equ. 3(3), 273-298 (1995)

[21] Cannarsa, P., Sinestrari, C.: Semiconcave Functions, Hamilton-Jacobi Equations, and Optimal Control. Progress in Nonlinear Differential Equations and Their Applications, vol. 58. Birkhäuser Boston Inc., Boston, MA (2004)

[22] Cannarsa, Piermarco, Soner, Halil Mete: On the singularities of the viscosity solutions to Hamilton-Jacobi-Bellman equations. Indiana Univ. Math. J. 36(3), 501-524 (1987)

[23] Cannarsa, Piermarco, Soner, Halil Mete: Generalized one-sided estimates for solutions of Hamilton-Jacobi equations and applications. Nonlinear Anal. Theory Methods Appl. 13(3), 305-323 (1989) 
[24] Capuzzo-Dolcetta, I., Ishii, Hitoshi: Approximate solutions of the Bellman equation of deterministic control theory. Appl. Math. Optim. 11(1), 161-181 (1984)

[25] Dong, Hongjie, Kim, Doyoon: Schauder estimates for a class of non-local elliptic equations. Discrete Contin. Dyn. Syst. 33(6), 2319-2347 (2013)

[26] Feleqi, Ermal: The derivation of ergodic mean field game equations for several populations of players. Dyn. Games Appl. 3(4), 523-536 (2013)

[27] Fleming, W.H., Mete Soner, H.: Controlled Markov Processes and Viscosity Solutions, Stochastic Modelling and Applied Probability, vol. 25, 2nd edn. Springer, New York (2006)

[28] Garroni, M.G., Menaldi, J.L.: Second Order Elliptic Integro-Differential Problems, CRC Research Notes in Mathematics, vol. 430. Chapman \& Hall, Boca Raton, FL (2002)

[29] Giga, Y., Goto, S., Ishii, H., Sato, M.-H.: Comparison principle and convexity preserving properties for singular degenerate parabolic equations on unbounded domains. Indiana Univ. Math. J. 40(2), 443-470 (1991)

[30] Gimbert, F., Lions, P.-L.: Existence and regularity results for solutions of secondorder, elliptic integro-differential operators. Ricerche Mat. 33(2), 315-358 (1984)

[31] Ishii, H., Lions, P.-L.: Viscosity solutions of fully nonlinear second-order elliptic partial differential equations. J. Differ. Equ. 83(1), 26-78 (1990)

[32] Jin, Tianling, Xiong, Jingang: Schauder estimates for solutions of linear parabolic integro-differential equations. Discrete Contin. Dyn. Syst. 35(12), 59775998 (2015)

[33] Jing, Shuai: Regularity properties of viscosity solutions of integro-partial differential equations of Hamilton-Jacobi-Bellman type. Stoch. Process. Appl. 123(2), 300-328 (2013)

[34] Kassmann, Moritz: A priori estimates for integro-differential operators with measurable kernels. Calc. Var. Partial Differ. Equ. 34(1), 1-21 (2009)

[35] Khrustalev, M.M.: Necessary and sufficient optimality conditions in the form of Bellman's equation. Doklady Akademii Nauk Russ. Acad. Sci. 242, 1023-1026 (1978)

[36] Kriventsov, Dennis: $C^{1, \alpha}$ interior regularity for nonlinear nonlocal elliptic equations with rough kernels. Commun. Partial Differ. Equ. 38(12), 2081-2106 (2013)

[37] Kruzhkov, S.N.: The cauchy problem in the large for certain non-linear first order differential equations. Doklady Akademii Nauk Russ. Acad. Sci. 132, 3639 (1960)

[38] Kruzhkov, Stanislav Nikolaevich: Generalized solutions of nonlinear equations of the first order with several variables. I. Matematicheskii Sbornik 112(3), 394-415 (1966) 
[39] Kružkov, S.N.: Generalized solutions of the Hamilton-Jacobi equations of eikonal type. I. Formulation of the problems; existence, uniqueness and stability theorems; some properties of the solutions. Sbornik: Mathematics 27(3), 406-446 (1975)

[40] Krylov, N.V.: Controlled Diffusion Processes, Stochastic Modelling and Applied Probability, vol. 14. Springer, Berlin (2009). (translated from the 1977 Russian original by A. B. Aries, reprint of the 1980 edition)

[41] Kulik, Alexey M: Some remarks on time-stretching differentiation for general lévy processes. Theory Stoch. Process. 7(23), 3-4 (2001)

[42] Kulik, O.M.: Malliavin calculus for Lévy processes with arbitrary Léivy measures. Teor. Imovīr. Mat. Stat. 72, 67-83 (2005)

[43] Kunita, H.: Stochastic differential equations based on Lévy processes and stochastic flows of diffeomorphisms. In: Real and Stochastic Analysis, Trends in Mathematics, pp. 305-373. Birkhäuser, Boston, MA (2004)

[44] Lara, Héctor Chang, Dávila, Gonzalo: Regularity for solutions of non local parabolic equations. Calc. Var. Partial Differ. Equ. 49(1-2), 139-172 (2014)

[45] Lara, Héctor Chang, Dávila, Gonzalo: $C^{\sigma, \alpha}$ estimates for concave, non-local parabolic equations with critical drift. J. Integral Equ. Appl. 28(3), 373-394 (2016)

[46] Lasry, Jean-Michel, Lions, Pierre-Louis: Jeux à champ moyen. I. Le cas stationnaire. C. R. Math. Acad. Sci. Paris 343(9), 619-625 (2006)

[47] Lasry, Jean-Michel, Lions, Pierre-Louis: Jeux à champ moyen. II. Horizon fini et contrôle optimal. C. R. Math. Acad. Sci. Paris 343(10), 679-684 (2006)

[48] Lasry, Jean-Michel, Lions, Pierre-Louis: Mean field games. Jpn. J. Math. 2(1), 229-260 (2007)

[49] Leonori, Tommaso, Peral, Ireneo, Primo, Ana, Soria, Fernando: Basic estimates for solutions of a class of nonlocal elliptic and parabolic equations. Discrete Contin. Dyn. Syst. 35(12), 6031-6068 (2015)

[50] Lindgren, Erik: Hölder estimates for viscosity solutions of equations of fractional p-Laplace type. NoDEA Nonlinear Differ. Equ. Appl. 23(5), 18 (2016)

[51] Lions, P.-L.: Optimal control of diffusion processes and Hamilton-JacobiBellman equations. III: Regularity of the optimal cost function. Nonlinear partial differential equations and their applications, Collège de France Seminar 19811982, vol. V, Research Notes in Mathematics, vol. 93, pp. 95-205 (1983)

[52] Lions, Pierre-Louis: Generalized Solutions of Hamilton-Jacobi Equations, vol. 69. Pitman, London (1982)

[53] Mou, Chenchen: Semiconcavity of viscosity solutions for a class of degenerate elliptic integro-differential equations in $\mathbb{R}^{n}$. Indiana Univ. Math. J. 65(6), 18911920 (2016) 
[54] Øksendal, Bernt, Sulem, Agnès: Applied Stochastic Control of Jump Diffusions. Universitext, 2nd edn. Springer, Berlin (2007)

[55] Pham, Huyên: Optimal stopping of controlled jump diffusion processes: a viscosity solution approach. J. Math. Syst. Estim. Control 8(1), 27 (1998). (electronic)

[56] Yong, J., Zhou, X.Y.: Stochastic Controls, Applications of Mathematics. Hamiltonian systems and HJB equations, vol. 43. Springer, New York (1999)

Ermal Feleqi

Cardiff School of Mathematics

Cardiff University

Senghennydd Road

Cardiff CF24 4AG

UK

e-mail: FeleqiE@cardiff.ac.uk

Received: 15 December 2014.

Accepted: 26 December 2018. 